\title{
Usagers et usages des livres numériques en lecture publique : l'exemple de Grenoble
}

\section{Emmanuel Brandl et Delphine Merrien}

\section{(2) OpenEdition}

1 Journals

Édition électronique

URL : http://journals.openedition.org/rbnu/289

DOI : 10.4000/rbnu.289

ISSN : 2679-6104

Éditeur

Bibliothèque nationale et universitaire de Strasbourg

\section{Édition imprimée}

Date de publication : 23 mai 2018

Pagination : $32-41$

ISSN : 2109-2761

\section{Référence électronique}

Emmanuel Brandl et Delphine Merrien, « Usagers et usages des livres numériques en lecture publique : l'exemple de Grenoble », La Revue de la BNU [En ligne], 17 | 2018, mis en ligne le 05 septembre 2018, consulté le 10 décembre 2020. URL : http://journals.openedition.org/rbnu/289 ; DOI : https://doi.org/10.4000/rbnu.289

\section{(c) (i) (8)}

La Revue de la BNU est mise à disposition selon les termes de la Licence Creative Commons Attribution - Pas d'Utilisation Commerciale - Partage dans les Mêmes Conditions 4.0 International. 
Printemps du livre de Grenoble

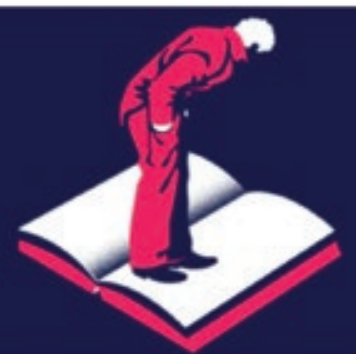

21-25 mars 2018

Bibliothèques musée et théâtre

Printemps du livre 2018

-

Découvrez les auteurs invités, en version numérique

Nouveautés (

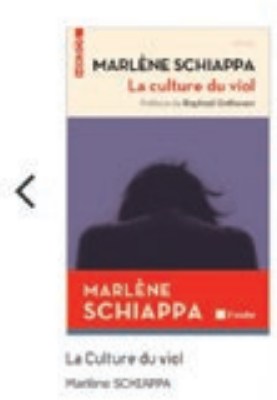

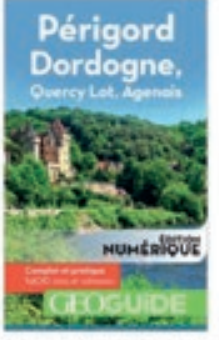

Ecopuide Prigoed Derdoget. OJercy Lot, Agenais

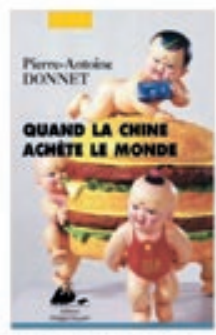

mande pereveremeaner

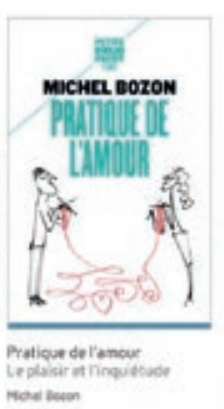

Woir le cotalogue ,

EAN ZIEQLER

Les murs les plus paissants

tombent

parkus

fissures

inis

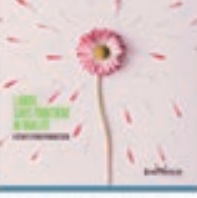

Lospirituelate do lo giene Dnesem Maes

visurets

Les murs ies plus puissentes Jen zave
Sélections de votre bibliothèque
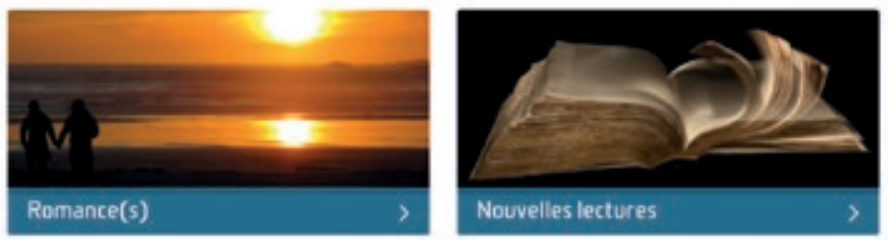

Vor the ,

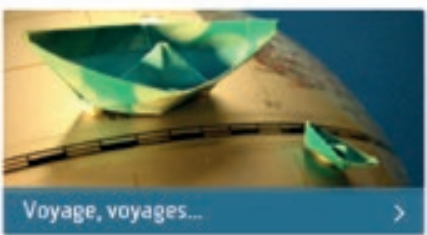




\section{USAGERS ET USAGES DES LIVRES NUMÉRIQUES EN LECTURE PUBLIQUE: L'EXEMPLE DE GRENOBLE}

PAR EMMANUEL BRANDL ET DELPHINE MERRIEN

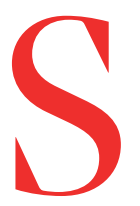

i, en 2016, $23 \%$ des bibliothèques municipales françaises proposaient des ressources numériques à leurs usagers, cette proportion passait à $82 \%$ pour les villes dépassant 70000 habitants ${ }^{1}$. Cependant, même si moins de la moitié de ces bibliothèques proposaient des ressources numériques incluant des livres numériques ${ }^{2}$, il reste que, d'après le Syndicat national de l'édition, « les bibliothèques représentent $4 \%$ des achats de livres [numériques] en France et ont une mission fondamentale de médiation pour l'élargissement du lectorat ${ }^{3}$.

C'est ainsi qu'apparaissent en 2010 le label «Bibliothèque numérique de référence " délivré par le ministère de la Culture et de la Communication afin d'inciter les bibliothèques à rendre accessibles en ligne les ressources numérisées, puis, en 2012, le projet «PNB » (Prêt numérique en bibliothèque) ${ }^{4}$. PNB est issu d'une initiative interprofessionnelle lancée par Dilicom (Réseau du livre) et soutenue par le Centre national du livre (CNL). Il consiste en un dispositif d'échange d'informations (données commerciales, métadonnées descriptives, juridiques et techniques, données de gestion : état des droits de consultation, statistiques, etc.) entre éditeurs, libraires et bibliothèques, dans le but de faciliter le développement d'offres de livres numériques accessibles via les bibliothèques publiques. Depuis le déploiement de ces dispositifs, et en dehors des données confidentielles propres aux établissements, les enquêtes et évaluations publiées à ce jour sur la thématique générale du livre numérique en bibliothèque ne font pourtant pas mention de données permettant de caractériser les usagers et leurs usages.
Nous n'insisterons pas ici sur les publications régulières du Baromètre des usages du livre numérique ${ }^{5}$, puisqu'elles ne portent pas sur la question du prêt en bibliothèques mais sur l'achat de livres numériques réalisé par des particuliers. Par ailleurs, l'évaluation de PNB publiée en 2016 par le ministère de la Culture ${ }^{6}$, de même que l'ouvrage Le livre numérique en bibliothèque de Laurent Soual récemment paru, ne livrent aucune donnée fine sur ce lectorat numérique.

Dans le même temps, l'augmentation et la diversification de l'offre numérique en bibliothèque ne peuvent qu'agir sur la demande et contribuer à en modifier les caractéristiques. De plus, les enjeux, économiques bien sûr, mais aussi symboliques, politiques et sociaux, liés à la question du livre numérique en bibliothèque ne peuvent faire l'économie d'une analyse du prêt de livres numériques dont PNB est un exemple (pas le seul certes, mais le seul coordonné au niveau national sur un grand nombre d'équipements ${ }^{7}$ ).

C'est dans ce contexte que la bibliothèque municipale de Grenoble (réseau de 12 bibliothèques) s'est associée à l'Enssib pour produire une étude qualitative et quantitative visant à caractériser les usagers et les usages de son service de prêt de livres numériques ${ }^{8}$ («Bibook »), lequel a ouvert au public au second semestre 2014. L'étude visait à établir un premier état des lieux du service huit mois après son ouverture, le corpus étant alors de 493 emprunteurs. 
CARACTÉRISATION DES USAGERS : DES PROFILS SOCIAUX PROPRES AUX PRATIQUES CULTURELLES ET DE LECTURE

\section{Une prédominance des professions et catégories socio-professionnelles supérieures}

Les logiques sociales qui président aux usages du service de prêt de livres numériques à Grenoble reproduisent celles généralement constatées pour les pratiques culturelles, et spécifiquement pour la lecture.

Ainsi, $56 \%$ de la population des usagers du service relèvent de ce qu'il est convenu d'appeler les PCS+ (professions et catégories socioprofessionnelles supérieures) - $34 \%$ pour les professions libérales/cadres supérieurs et $22 \%$ pour les professions intermédiaires, alors que ces deux catégories cumulées représentent $23 \%$ environ de la population française générale ${ }^{9}$.

Cette proportion élevée s'explique notamment par le fait que ces catégories dites « moyennes et supérieures » bénéficient d'un niveau de formation plus élevé que celui des autres catégories sociales, d'une latitude plus importante que celle des employés pour gérer leur temps, et d'un rapport à la lecture davantage caractérisé par une forme de «bonne volonté culturelle » ${ }^{10}$.

Pour ces catégories d'usagers, la bibliothèque constitue un espace - réel et/ou virtuel - culturel, contrairement aux catégories sociales dites "défavorisées ", pour lesquelles la bibliothèque est d'abord un lieu (de fait plutôt physique) de convivialité.

\section{Une féminisation des emprunts}

Les femmes représentent $70 \%$ des usagers du service, contre $51,6 \%$ de la population nationale ${ }^{11}$. La part majoritaire des femmes se vérifie quelle que soit la catégorie socio-professionnelle concernée.

Notre résultat est légèrement supérieur aux taux constatés à propos du prêt imprimé par Bruno Maresca, Christophe Evans et Françoise Gaudet dans leur étude sur le « rayonnement des bibliothèques municipales » en 2007 : «Au sein du public des bibliothèques municipales, les femmes dominent largement : $60 \%$ des usagers sont des femmes $"{ }^{12}$.

Toutefois, selon la catégorie socio-professionnelle, l'écart entre hommes et femmes varie de façon non négligeable : le ratio est de 1 à 2 , voire de 1 à 4 . De ce point de vue, nos données ne recouvrent pas les caractéristiques démographiques habituellement décrites des populations des emprunteurs-lecteurs de livres numériques ${ }^{13}$ (homme trentenaire / femme quinquagénaire). Elles recouvrent en revanche les caractéristiques de la population générale des bibliothèques municipales ${ }^{14}$.

\section{FIGURE 1}

Répartition des emprunteurs selon le sexe et la catégorie socio-professionnelle

Femmes

Hommes

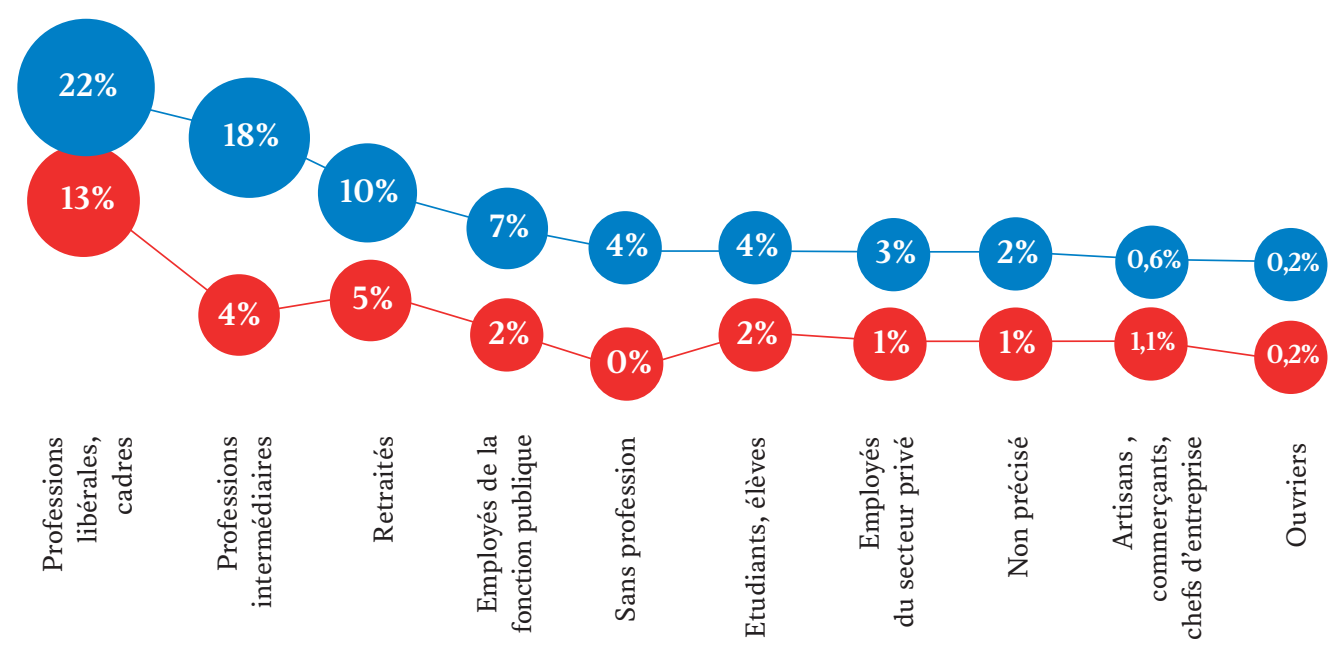




\section{Un vieillissement du lectorat}

La classe d'âge la plus représentée parmi les usagers du service de prêt de livres numériques à Grenoble est celle des 50-64 ans, les "plus de 50 ans " représentant près de la moitié de notre corpus (48\%). Les moins de 25 ans en représentent quant à eux $9 \%$, et les $25-29$ ans $5 \%$. Les moins de 25 ans comptent une majorité d'étudiants et élèves, et les 25-29 ans comptent une majorité d'actifs (ce sont de " jeunes actifs ») des PCS supérieures (pour $65 \%$ ).

La moyenne d'âge des femmes est de 47 ans, celle des hommes de 51 ans, ce qui porte la moyenne d'âge globale des usagers du service à 48 ans. C'est un résultat notable quand on sait que la moyenne d'âge des acheteurs de livres numériques est de moins de 35 ans $^{15}$.

Nos résultats s'inscrivent de fait dans un processus général d'évolution des pratiques culturelles, comme la fréquentation du spectacle vivant - et notamment les concerts de musique classique -, mais aussi le cinéma en salle ${ }^{16}$. En effet, ce vieillissement mesuré des usagers est issu de l'accroissement de la proportion des seniors dans la population française, de l'évolution de leurs modes de loisirs - davantage tournés vers les sorties -, mais aussi, parfois, de la désaffection des jeunes pour les pratiques culturelles, et en particulier la lecture d'ouvrages.

\section{Une origine géographique majoritairement locale}

Les usagers du service de prêt de livres numériques de Grenoble sont essentiellement locaux (Isérois à $96 \%$ ) et urbains. Ils viennent d'abord de Grenoble et de sa proche banlieue (à $83 \%$ ).

Cela signifie que ce service en ligne reste majoritairement utilisé par des personnes qui habitent à proximité des bibliothèques concernées, et qui - très certainement - les fréquentent. De plus, comme le rappelle Jean-François Hersent ${ }^{17}$, dans les représentations sociales des individus, la bibliothèque reste l'équipement culturel « de proximité » (sociale et géographique) par excellence : la bibliothèque municipale est perçue comme un lieu familier, proche aussi bien du domicile que du lieu de travail ${ }^{18}$.
FIGURE 2

Répartition géographique des emprunteurs

- Grenoble

- Territoire communauté d'agglomération (sans grenoble)

Isère

Rhône-Alpes (hors Isère)

Hors région

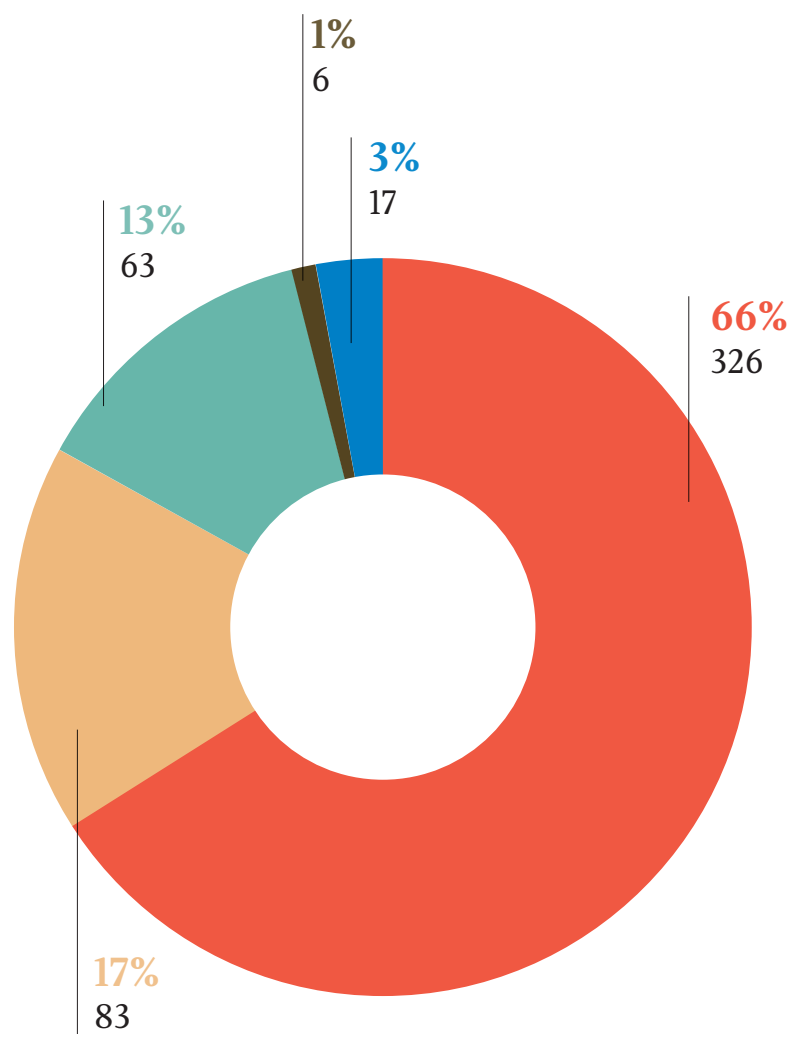




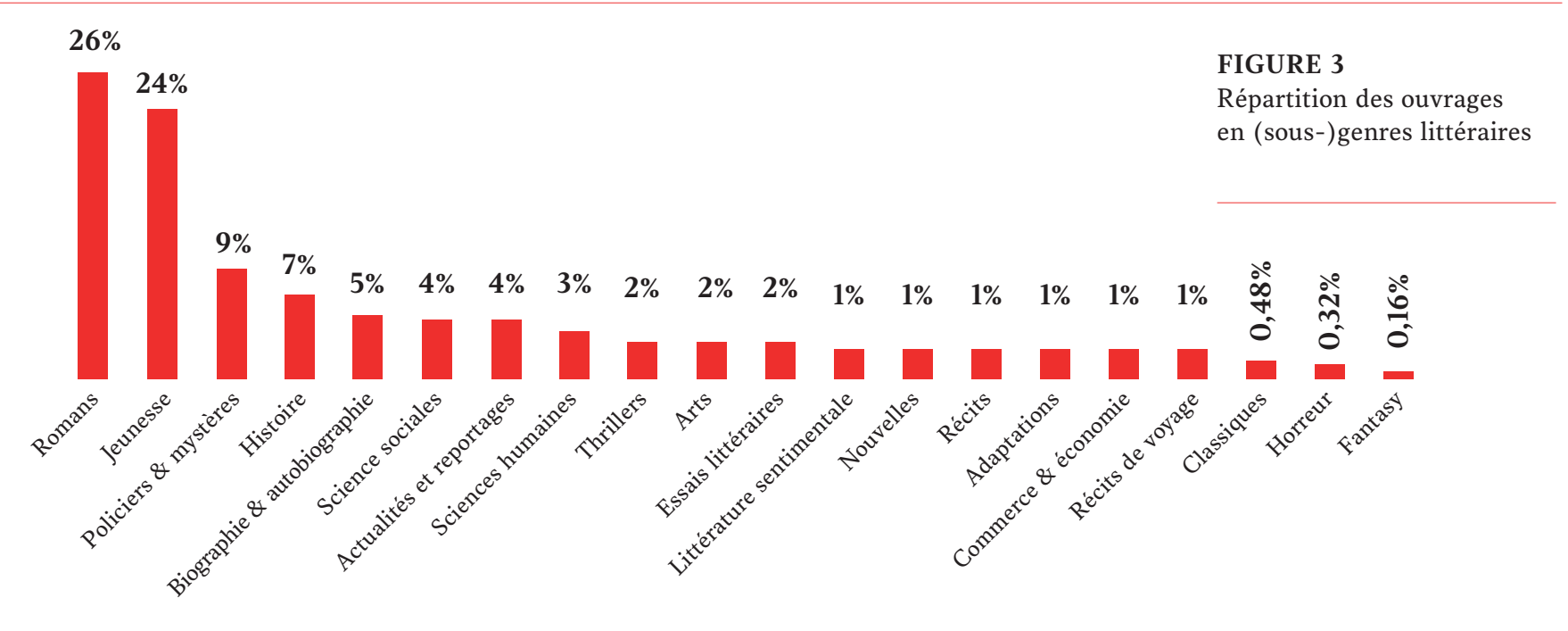

\section{Caractérisation du catalogue : l'offre de livres électroniques à Grenoble}

En mars 2015, le catalogue de livres électroniques de la Bibliothèque municipale de Grenoble (BMG) proposait 611 titres différents au téléchargement. Ces titres étaient répartis entre 51 éditeurs et 409 auteurs.

À partir des données de répartition des genres fournies par la BMG, les titres ont été regroupés en deux grands ensembles : "fiction » (romans, jeunesse, policiers et mystères, thrillers, littérature sentimentale, nouvelles, récits, adaptations, récits de voyage, classiques, horreur, fantasy) et "non fiction » (histoire, biographie et autobiographie, sciences sociales, sciences humaines, arts, essais littéraires, actualités et reportages, commerce et économie). Le catalogue comprend ainsi $65 \%$ de livres numériques dits de "fiction", et une nette prédominance du genre "roman » et des ouvrages dits « jeunesse » apparaît, puisque ces deux catégories représentent à elles seules $50 \%$ du catalogue.

La répartition des auteurs, titres et téléchargements par maison d'édition révèle une prédominance de la maison d'édition Gallimard, qui représente $26 \%$ des auteurs, $24 \%$ des titres et $41 \%$ des téléchargements.

\section{CARACTÉRISATION DES USAGES : USAGES CONFORTÉS ET DIVERSIFIÉS DANS UNE VOLUMÉTRIE CONTENUE}

\section{Une évolution favorable des emprunts}

Contrairement aux autres données de l'étude menée en 2015, la figure 4 porte sur une période plus étendue. En effet, les données ont continué à être collectées par Grenoble et transmises à l'Enssib, ce qui a permis de renseigner certains phénomènes sur une durée plus longue et de produire le Baromètre Enssib du livre numérique en bibliothèque, dont la première édition porte sur les données $2016^{19}$.

Elle permet de mettre en évidence deux

phénomènes majeurs :

- une nette augmentation des téléchargements entre septembre et novembre 2014, ce qui tient à l'effet de nouveauté du dispositif, mais aussi à un travail de médiation réussi par les bibliothécaires de Grenoble ;

- une augmentation légère mais continue sur le reste de la période, avec un saut quantitatif au mois de juillet 2015. S'il est délicat en l'état actuel des données d'interpréter ce résultat, nous pouvons émettre l'hypothèse d'un effet des vacances scolaires (on emprunte des livres qu'on lira en vacances), phénomène qui peut être renforcé par l'augmentation concomitante de l'offre de livres numériques (effet de nouveauté de l'offre). 
L'intérêt pour Bibook ne s'est donc pas démenti avec le temps. Au contraire, il s'en trouve progressivement renforcé. Car dans le même sens, nous constatons une augmentation mensuelle régulière des emprunts entre 2014 et 2015 (augmentation mensuelle moyenne de 15,7\%), le plus fort taux de téléchargement, c'est-à-dire le rapport entre le nombre d'individus et le nombre de téléchargements effectués, étant au moment de l'enquête enregistré chez les « 65 ans et plus ».

\section{Le numérique comme medium facilitateur d'une diversité culturelle?}

L'analyse de la répartition des emprunts par maison d'édition et des "visites " de maisons d'édition différentes (sans emprunt, mais à la façon dont on « visite " un site par curiosité), montre que toutes les catégories socio-professionnelles font l'objet d'une fidélité ou d'un attachement fort à une maison d'édition en particulier. Cependant, les PCS supérieures font preuve d'une plus grande " curiosité littéraire " que les autres, bien que toutes les PCS soient en réalité concernées par une forme plus ou moins prononcée d'éclectisme éditorial.

Nos résultats rejoignent une tendance générale dans les pratiques culturelles, celle d'une diversification des pratiques ${ }^{20}$, et permettent de penser que le numérique peut être un médium facilitateur de la diversification des pratiques d'emprunt et de lecture pour les usagers des bibliothèques : peut-être parce qu'il permet par exemple de ne pas avoir à se déplacer physiquement dans la bibliothèque pour explorer le catalogue à disposition.

\section{FIGURE 5}

Nombre de maisons d'édition « cliquées » par PCS

(avec ou sans emprunt)

\begin{tabular}{|c|c|}
\hline Professions libérales / cadres supérieurs & 44 \\
\hline Professions intermédiaires & 35 \\
\hline Retraités & 33 \\
\hline Étudiants, élèves & 25 \\
\hline Employés de la fonction publique & 23 \\
\hline Artisans / commerçants / chefs d'entreprise & 22 \\
\hline Employés du secteur privé & 21 \\
\hline Non précisé & 21 \\
\hline Sans profession & 20 \\
\hline Ouvriers & 3 \\
\hline
\end{tabular}

Si l'impact sur la démocratisation culturelle d'une offre de livres numériques dans une bibliothèque de lecture publique reste limité, il ressort en revanche que ce medium, à cet endroit, peut remplir un rôle de brouillage de ce qu'il est convenu d'appeler « les effets de la légitimité culturelle ».

Pour cela, il faut qu'existe et soit entretenue une relation entre l'offre et la demande de livres numériques, et donc penser au niveau local les motivations des usagers à s'aventurer sur d'autres terrains littéraires que ceux qu'ils fréquentent habituellement, afin de répondre au mieux à cette " curiosité littéraire " que nous constatons, voire de l'encourager, dans une logique de recommandation et de sérendipité.

\section{FIGURE 4}

Une évolution favorable de la consommmation dans le temps

\footnotetext{
$\longrightarrow$ Emprunts mensuels

— Évolution mensuelle du catalogue

— Évolution mensuelle du nombre de nouveaux comptes Bibook
}

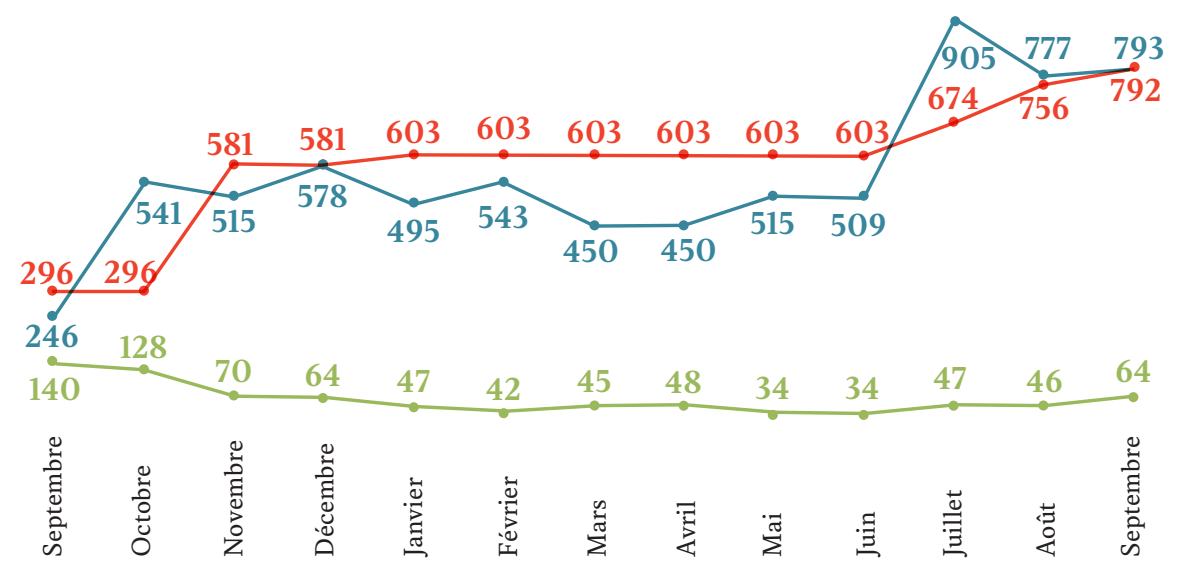


Cela revient à définir une politique documentaire numérique à partir d'une meilleure connaissance des attentes des usagers, sans se limiter à l'analyse des usages effectifs (c'est-à-dire des emprunts comptabilisés), et en portant une attention particulière aux logiques d'exploration du catalogue.

\section{Des anciens abonnés qui s'inscrivent plus tôt que les autres...}

Si l'on analyse en détail les inscriptions au service et que l'on cherche à caractériser les variables qui déterminent le moment de cette inscription, on s'aperçoit bien vite que les variables socio-démographiques ne sont pas discriminantes dans l'appétence que les individus du corpus ont eue pour le service Bibook à son ouverture.

On constate en revanche que ce sont les plus anciens abonnés à la bibliothèque qui s'inscrivent en majorité le plus tôt à ce service. Les catégories «testeurs » (inscrits au service entre septembre et décembre 2014), " explorateurs» (inscrits au service entre janvier et mars 2015) et " découvreurs " (inscrits au service entre avril et juin 2015) se distinguent ainsi par leur ancienneté et non par quelque variable socio-démographique.
L'inscription à ce service semble ainsi répondre à un effet cumulatif d'usages : plus une personne fréquente une bibliothèque, plus elle gagne en aisance, utilise des services diversifiés et connaît le personnel, avec lequel elle entre plus aisément en interaction. C'est dans ce cas précis que les médiations mises en place dans les bibliothèques ont le plus d'impact.

\section{- Numérique et papier : intensification et migration des usages}

Les études qui portent sur les usages du livre numérique restituent toutes que les usagers de livres numériques sont aussi de "grands lecteurs" (de livres imprimés). Récemment, une étude menée au sein des publics de la médiathèque de Meyzieu (Rhône-Alpes) rendait compte du fait que « $51 \%$ (des personnes ayant répondu au questionnaire sur l'usage des liseuses) déclarent lire plus de 20 livres par an, et $26 \%$ déclarent lire entre 10 et 19 livres par an ${ }^{21}$.

En l'occurrence, nos résultats invitent à nuancer cette unanimité. En effet, si $21 \%$ des emprunteurs actifs ont emprunté " 20 livres imprimés et plus » sur l'année, 38 \% d'entre eux n'ont emprunté aucun livre papier. Pourtant, le profil sociologique de ces différents usagers est identique. On ne trouve donc aucun élément d'explication des différences de comportement dans les facteurs sociaux.

\section{FIGURE 6}

Testeurs, explorateurs et découvreurs du service

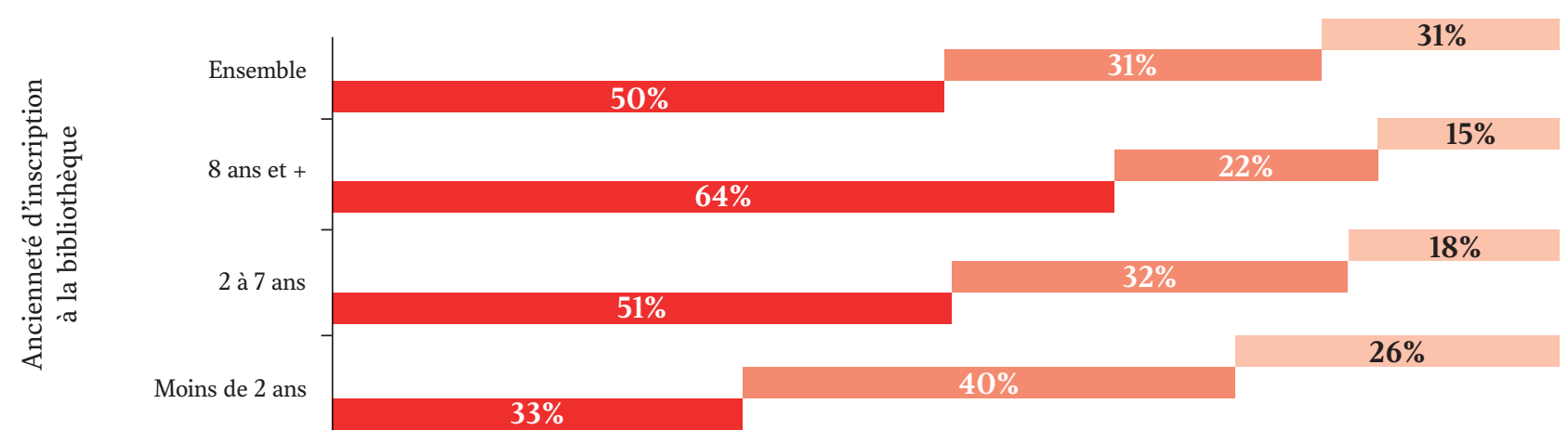


Date de parution

\section{LANGUE \\ Francais (1056) \\ Angla's (38) \\ DATE DE PARUTION \\ 3 derniers mols (18) \\ 6 derniers mois (42) \\ 12 derniers mois (103) \\ Moins de 3 ans (533)}

\section{PUBLIC}

Adulte (938)

Jeunesse (145)

\section{CATÉborie}

Action (62.7)

Booman (250)

Jeunesse (143)

Uttirature sertimertale (S6)

Poliders \& Mystives (53)

Solence-fiction (3)

Historiase (35)

Rlat (35)

Thrillers (34)

Thematiques (33)

Fantasy (29)

Nouvelies (21)

Bande dessinke \& Roman

graphique (10)

Arthologies (8)

Cassiques (6)

Adaptations (6)

Fantastique (6)

Documentaires (S41)

Solences sciales (163)

Biograchie \& Autobicgraphin (113)

Histaine (86)

Actualnt et Reportages (82)

soences humeines (67)

Sante \& Ve quobdienne (55)

Nature, bisin et sports (41)

Solences et Techrieues (36)

Ats (39)

Ve quotidenne (32)

Histaire por pons (31)

Colections ittidraies (30)

Solences (30)

Commerce $\mathrm{s}$ toonomie (28)

Refierence (27)

Europe (20)

Noture (19)

Redigion (16)

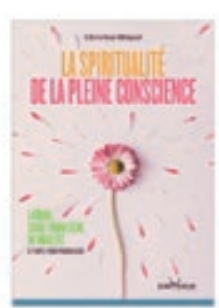

La spiritualite de la pleine

Oristian Mose

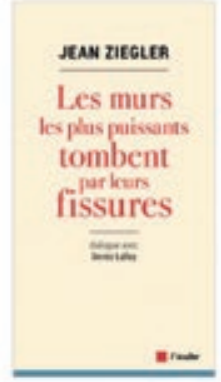

Lesmurs les plus puissants Lembent perieurs then zeaek.

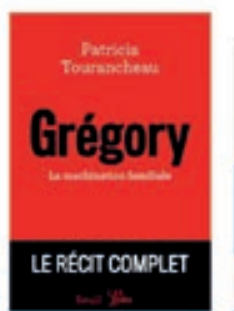

Grêgery-La mechinotion familisie

Neide turenchesu

rede roweses

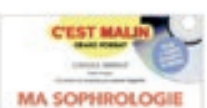

Ma sopurotogit ANTSTRESS
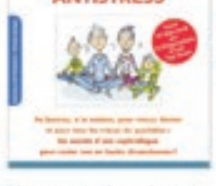

Mu saphrologie antistress: Cest malin

Aubureas, a is maison. peser minur dermir en sour tevilestracas dy

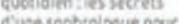
duse sophrotoque pas cictenstances!

cacie semat

\begin{tabular}{|l|}
\hline Les Spectateun \\
\hline NATHALIE \\
AZOULAI
\end{tabular}

Lnspectaters Netrane nowat



Pres guide d rusoge des percents qui trourend d juste titue que intent mescice venthe
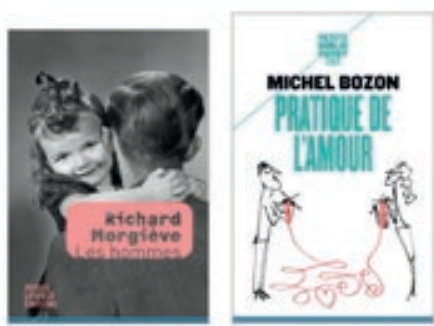

Les hommes Rodurd Morplewe

Pratique de ramour Lepluisir et ringuletude matha Borom

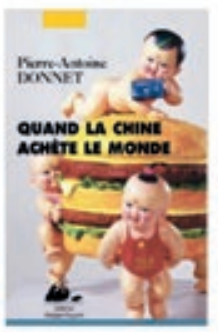

Quand la Chine achete le mende

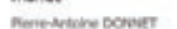

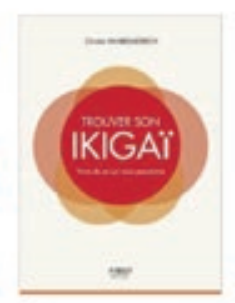

Trouver son 1kiga!

Orise venoaperassor

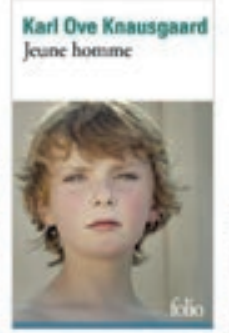

Ven combet (feme 3) Jeune hemme

cor one knompend



100 questions syr

Mitheimer

Merc veawy, Vooine owr.

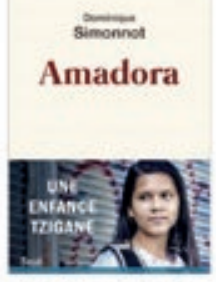
tzigane

Dominen Snover

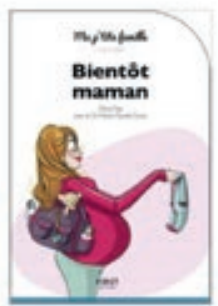

Biencele maman, be eation ona tosa
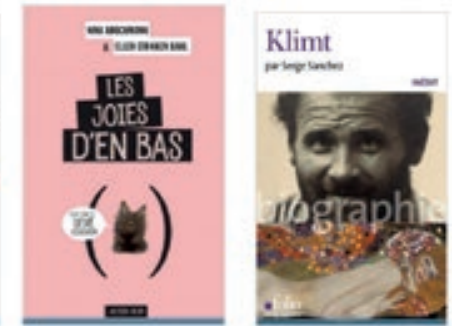

Les Joies den bas

Tout sur le sese feminin

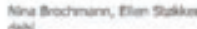

Klimt

Singersincter

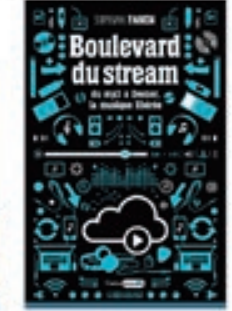

Beulereed de Stream seoran tom 
En fait, pour les $21 \%$ d'usagers qui ont emprunté « 20 livres imprimés et plus », le livre numérique arrive en complémentarité du livre papier et indique une intensification de la pratique. Cette catégorie est donc aussi celle des " grands lecteurs », ces « dogmatiques de la lecture $»$.

Mais a contrario, pour les $38 \%$ d'usagers qui n'empruntent aucun livre imprimé, le livre numérique est exclusif d'un emprunt papier. Ils sont alors définis comme des " migrants ", c'est-à-dire des lecteurs de livres papier qui, notamment pour des raisons pratiques (comme le fait d'habiter loin d'un centre urbain, ici Grenoble), sont passés au « tout numérique » ${ }^{22}$.

\section{Typologie des usages : une prédominance des petits lecteurs}

L'analyse quantitative des pratiques d'emprunt (c'est-à-dire du nombre de livres téléchargés dans l'année) permet de dégager trois grands types d'emprunteurs.

- Les «faibles emprunteurs-lecteurs », qui ont emprunté entre 1 et 9 livres numériques sur la période considérée, représentent une très large majorité des emprunteurs (90\%), mais n'ont réalisé que $65 \%$ du total des emprunts : davantage de personnes empruntent globalement moins.

- Les emprunteurs-lecteurs " moyens » empruntent entre 10 et 19 livres numériques sur la période considérée : ils représentent $8,7 \%$ des emprunteurs et réalisent $27,5 \%$ des emprunts.

\section{FIGURE 7}

Répartition des téléchargements :

une prédominance des petits emprunteurs
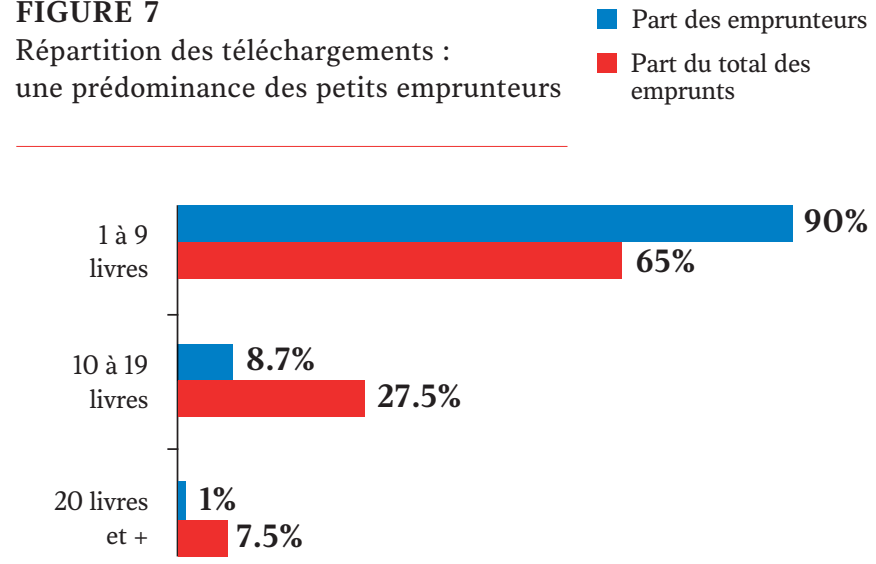

- Les " gros emprunteurs-lecteurs ", qui empruntent 20 livres numériques et plus sur la période considérée, représentent $1,3 \%$ des emprunteurs et réalisent $7,5 \%$ des emprunts.

Ici, l'enquête livre deux enseignements intéressants à retenir :

- Les variables qui semblent faire la différence entre ces trois catégories d'usagers sont celles du sexe et de l'âge, lequel permet de distinguer significativement les deux dernières catégories de la première.

- Si le genre littéraire "fiction " est dominant dans les emprunts comptabilisés, il ressort clairement qu'il n'est pas exclusif d'emprunts non fictionnels, et que c'est d'abord une logique de double emprunt (fiction / non fiction) qui domine la réalité des usages du service de prêt de livres numériques de Grenoble.

\section{Enseignements et prolongements}

Il nous a été possible de caractériser le profil «type » de l'emprunteur (de l'emprunteuse...) de livres numériques : il s'agit d'une femme d'une cinquantaine d'années, appartenant aux PCS supérieures, habitant en zone urbaine (et péri-urbaine) et lisant de la fiction.

La dimension exploratoire du catalogue numérique apparaît comme un aspect à prendre en compte dans la politique documentaire numérique d'une bibliothèque. Sur un plan bibliothéconomique, une étude comme celle-ci a notamment contribué à rapprocher l'offre éditoriale de la bibliothèque municipale de Grenoble de la demande de ses lecteurs, en confirmant certaines hypothèses de départ, notamment « privilégier les nouveautés en fiction et la diversité des titres plutôt que multiplier les exemplaires ${ }^{23}$.

Dans le prolongement d'une telle approche, et au-delà de la bibliothèque en tant que telle, il reste à mieux comprendre les processus et matrices de socialisation par lesquels se diffuse et s'acquiert la « culture de la lecture (sur support) numérique ", notamment au sein de la famille. S'ouvre alors un questionnement sur les modalités concrètes par lesquelles les lecteurs sur support numérique en sont venus à lire et à aimer lire sur ces supports, et la diffusion qu'ils en font euxmêmes. 
1- Soual, L., Le livre numérique en bibliothèque : état des lieux et perspectives, Paris, Éditions du Cercle de la librairie, 2015

2- Ibid.

3- Page « Bibliothèques et numérique » sur https://www.sne.fr/ numerique-2/bibliotheques-numeriques/ (page consultée le 31/01/2018).

4- Le service PNB a été déployé dès 2012 dans quatre établissements, Aulnay-sous-Bois, Grenoble, Montpellier et Levallois, lesquels ont été rejoints par la Ville de Paris au premier semestre 2015.

5- Nous renvoyons le lecteur aux différents baromètres publiés depuis 2012 par la Sofia (Société française des intérêts des auteurs de l'écrit), le SNE (Syndicat national de l'édition) et la SGDL (Société des gens de lettres), et produits par OpinionWay. Disponibles en ligne : https://www.sne.fr/numerique-2/barometre-sur-les-usages-dulivre-numerique/ (page consultée le 31/01/2018).

6- Éléments d'évaluation du dispositif Prêt numérique en bibliothèque. Rapport. Ministère de la Culture et de la Communication, mars 2016. Disponible en ligne : http://www.enssib.fr/bibliotheque-numerique/ documents/66019-elements-d-evaluation-du-dispositif-pret-numeriqueen-bibliotheque.pdf (consulté le 31/01/2018).

7- $\mathrm{Au} 1^{\text {er }}$ mars 2017, 117 bibliothèques ou réseaux de bibliothèques sont en production, c'est-à-dire raccordés techniquement au service $\mathrm{PNB}$ et en mesure de faire des acquisitions auprès d'un libraire, et 86 bibliothèques sont émettrices de prêts. Cf. Éléments d'évaluation du dispositif Prêt numérique en bibliothèque, op. cit.

8- Le rapport complet (Brandl, E., Bibook à son lancement : quels usagers, quels usages ? Rapport d'étude, Enssib, 2016) est disponible en ligne : http://www.enssib.fr/bibliotheque-numerique/documents/67726-bibooka-son-lancement-quels-usagers-quels-usages.pdf.

9- Tableau POP6 V2 - Âge regroupé - sexe : ensemble. In Évolution et structure de la population en 2013. France entière. Disponible en ligne : https://www.insee.fr/fr/statistiques/2020211? sommaire =2106142\&geo=FE-1 (page consultée le 31/01/2018).

10- Bourdieu, P., La distinction. Critique sociale du jugement, Paris, Minuit, 1979

11- Tableau POP1A - Population par sexe et âge regroupés. In Évolution et structure de la population en 2013. France entière. Disponible en ligne : https://www.insee.fr/fr/ statistiques $/ 2020197$ ? sommaire $=2106142 \&$ geo $=F E-1$ (page consultée le 31/01/2018).

12- Maresca, B. et al., Les bibliothèques municipales après le tournant Internet. Attractivité, fréquentation et devenir, Paris, Éditions de la Bibliothèque publique d'information/Centre Pompidou, 2007, p. 78. Disponible en ligne : https://books.google.fr/ books/about/Les_biblioth\%C3\%A8ques_municipales_en_France. $h t m l$ ? id $=D N P 5 D A A A Q B A J \&$ source $=k p \_c o v e r \& r e d i r \_e s c=y$ (page consultée le 31/01/2018).

13- Voir par exemple Paquienseguy, F., Usages et consommation d'e-books en France. Bilan des études françaises disponibles. Séminaire Ebook AN2 : Liseuses et lecteurs, 2013, et Baromètre des usages du livre numérique. Sofia, SNE, SGDL, vague 3, février 2013.

14- Bertrand, A.-M., Les bibliothèques municipales et leurs publics. Pratiques ordinaires de la culture, Éditions de la Bibliothèque publique d'information/Centre Pompidou, 2001, p. 29. Disponible en ligne http://books.openedition.org/bibpompidou/238?lang=fr (page consultée le 31/01/2018).
15- Baromètre des usages du livre numérique, op. cit.

16- Donnat, O., « Les pratiques culturelles des Français à l'ère numérique. Éléments de synthèse 1997-2008 », in Culture-études, 2009-5. Disponible en ligne : http://www.pratiquesculturelles.culture. gouv.fr/doc/08synthese.pdf (page consultée le 31/01/2018).

17- Hersent, J.-F., Sociologie de la lecture en France : état des lieux (essai de synthèse à partir des travaux de recherche menés en France), DLL, juin 2000. Disponible en ligne : www.culture.gouv.fr/culture/dll/sociolog.rtf (page consultée le 31/01/2018)

18- Pour plus de précisions, voir Maresca, B. et al., op. cit.

19- Disponible en ligne : http://www.enssib.fr/bibliotheque-numerique/ documents/67995-barometre-enssib-2017-usages-du-service-de-pretde-livres-numeriques-de-la-bibliotheque-municipale-de-grenoble.pdf (page consultée le 26/02/2018).

20- Voir par exemple Donnat, O., Les Français face à la culture. De l'exclusion à l'éclectisme, Paris, La Découverte, 1994 ; Lahire, B., La culture des individus. Dissonances culturelles et distinction de soi, Paris, La Découverte, 2004.

21- Verdi, M., Résultats du questionnaire sur le prêt de liseuses Médiathèque de Meyzieu. Enssib, 2015. Document préparatoire à Verdi, M., Le numérique et le lecteur, retour du nomade. Une enquête dans les médiathèques en Auvergne-Rhône-Alpes, Presses de l'Enssib (collection La Numérique), 2017. Disponible en ligne : http://www. enssib.fr/sites/www/files/documents/presses-enssib/ebooks/verdi_lenumerique-et-le-lecteur.pdf (page consultée le 31/01/2018).

22- Voir Paquienseguy, F., Le lectorat numérique aujourd'hui : pratiques et usages, Paris, Éditions des archives contemporaines, 2015, p. 71 et suiv.

23- Brigant, A., Le Torrec, M. et Hatt, G., « Préface », in Brandl, E., Bibook à son lancement : quels usagers, quels usages? Rapport d'étude, Enssib, 2016, op. cit. 